\title{
Methicillin-resistant Staphylococcus aureus screening is important for surgeons
}

\author{
II-Kwang Hyun ${ }^{1, *}$, Pyoung Jae Park ${ }^{1,2, *}$, Dawon Park ${ }^{1,3}$, Sae Byeol Choi ${ }^{1,2}$, Hyung Joon Han ${ }^{1,4}$, \\ Tae-Jin Song ${ }^{1,4}$, Cheol-Woong Jung ${ }^{1,3}$, and Wan-Bae Kim ${ }^{1,2}$
}

\begin{abstract}
${ }^{1}$ Department of Surgery, Korea University College of Medicine, ${ }^{2}$ Department of Surgery, Korea University Guro Hospital, ${ }^{3}$ Department of Surgery, Korea University Anam Hospital, Seoul, ${ }^{4}$ Division of Hepatobiliopancreas and Transplant Surgery, Department of Surgery, Korea University Ansan Hospital, Ansan, Korea
\end{abstract}

\begin{abstract}
Backgrounds/Aims: Perioperative surgical site infection (SSI) remains a morbid complication even in successful surgical procedures. We encountered an unusual experience of a methicillin-resistant Staphylococcus aureus (MRSA)-related SSI outbreak in our hospital; therefore, we conducted an epidemiologic analysis to determine the origin of SSIs due to MRSA. Methods: Among 102 consecutive patients who underwent hepatobiliopancreatic operations, SSIs occurred in eight cases. Infection surveillance regarding the operative environment was carried out. We analyzed the possible risk factors for this infectious outbreak in our institution. Results: Patients with $S S I$ tended to be older $(p=0.293)$, had variable operation fields $(p=0.020)$, more cancer-related operation $(p=0.003)$, less laparoscopic surgery $(p=0.007)$, performed in operation room $1(p=0.004)$, prolonged operation time $(p<0.001)$ and had longer hospital stays $(p=0.002)$. After propensity score (PS) matching, there was the only significant difference in the participation of surgeon $D$ as a second assistant $(p=0.001)$ between the SSI and non-SSI group. After PS matching, surgeon $\mathrm{D}$ as a second assistant was the only significant risk factor for MRSA SSI in the univariate $(p=0.001)$ and multivariate analysis $(p=0.004$, hazard ratio $=25.088,95 \%$ confidence interval=2.759-228.149). Conclusions: Outbreak of SSIs occurred due to transmission of MRSA from a surgeon to patients despite the standard regulation of infection control. These SSIs were associated with an excessive incidence of surgeon's nasal and hand carriage of the MRSA strain identified in the surgeon via cultures. We recommend the preoperative regular nasal and hand screening for MRSA among surgeons. (Ann Hepatobiliary Pancreat Surg 2019;23:265-273)
\end{abstract}

Key Words: Surgical site infection; Methicillin-resistant Staphylococcus aureus; Screening

\section{INTRODUCTION}

Perioperative surgical site infection (SSI) remains a morbid complication even in successful surgical procedures. Staphylococcus aureus is the most common causative pathogen in SSIs and is a common risk factor of healthcare worker-related morbidity and mortality. ${ }^{1}$ Moreover, S. aureus accounts for a third of all SSIs according to the National Healthcare Safety Network report. ${ }^{2}$ In particular, methicillin-resistant $S$. aureus (MRSA) has been an important causative pathogen of global healthcare and community-acquired infections. ${ }^{3,4}$ The primary route of MRSA transmission is from patients to patients, from the environment to the patient, or even contact with the healthcare workers' hands. ${ }^{5}$ The most important transmission route usually involves contaminated hands. Microorganisms from the hands of the surgeon, organisms found in the operating room, or organisms from other surgical staff members are often causes of SSIs. ${ }^{6}$

Healthcare workers are expected to play an important role in MRSA transmission because the average rate of MRSA colonization among healthcare workers has been estimated at $4.6-6 \%{ }^{7,8}$ A decrease in the incidence of SSI due to $S$. aureus screening and decolonization has directly

Received: March 7, 2019; Revised: April 22, 2019; Accepted: April 30, 2019

Corresponding author: Hyung Joon Han

Division of Hepatobiliopancreas and Transplant Surgery, Department of Surgery, Korea University Ansan Hospital, 123 Jeokgeum-ro, Ansan 15355, Korea

Tel: +82-31-412-4826, Fax: +82-31-412-4829, E-mail: hjhan@korea.ac.kr

*Il-Kwang Hyun and Pyoung Jae Park contributed equally to this manuscript.

Copyright (C) 2019 by The Korean Association of Hepato-Biliary-Pancreatic Surgery

This is an Open Access article distributed under the terms of the Creative Commons Attribution Non-Commercial License (http://creativecommons.org/ licenses/by-nc/4.0) which permits unrestricted non-commercial use, distribution, and reproduction in any medium, provided the original work is properly cited. Annals of Hepato-Biliary-Pancreatic Surgery • pISSN: 2508-5778 - elSSN: 2508-5859 
attention towards preoperative $S$. aureus carriage. ${ }^{9}$ Healthcare workers have been known as an important source of SSIs, such as SSIs originating from a pair of perforated surgical gloves of a surgeon harboring known colonies of $S$. aureus in his/her nose. Air-borne intraoperative infection is another common transmission pathway. A previous study reported a case of postoperative SSI due to S. aureus disseminated via droplets from an operating room staff member with eczema, who was later identified as the disperser of the outbreak strain. ${ }^{10}$ A systematic review showed that 11 out of 191 nosocomial outbreaks of MRSA might have been caused by healthcare workers. Asymptomatic carriers were thought to be the cause in 3 of these SSI outbreaks. ${ }^{11}$

We encountered an unusual experience of a MRSA-related SSI outbreak in the hepatobiliopancreatic surgery division in our institution; we therefore performed an epidemiologic analysis to identify the origin of this outbreak. The aim of this study was to determine the origin of MRSA that was responsible for the SSI outbreaks.

\section{MATERIALS AND METHODS}

\section{Study design}

This was an observational, retrospective, and epidemiologic study conducted at single center, Korea University Medical Center. Between January and February 2009, among 102 consecutive patients who underwent hepatobiliopancreas operations, SSIs occurred in $8(7.8 \%)$ patients. An infection surveillance that involved patient characteristics, operative sterilization status, operation type, operation class, operation field, medical staff status, and in-hospital factors was carried out by a practitioner associated with the infectious disease division of the internal medicine department. Diagnosis of SSI was based on clinical findings, including redness, tenderness, and pus discharge of the operative wound, and identification of $>10^{5}$ organisms in the surgical sites. Then, the SSIs were classified as a superficial wound infection, deep wound infection, or intra-abdominal organ/space infection according to criteria from the Centers for Disease Control and Prevention (CDC). ${ }^{12}$ We compared the perioperative characteristics between the patients with the SSI and those without SSI. We then attempted to identify the possible risk factors of this infectious outbreak within our institution.
We divided hospital staff members into surgeons, first assistants, and second assistants according to the intraoperative role in the surgical field. Three surgeons (from A to C) or eight assistants (from B to I) participated in the operations and some operations were performed without an assistant. We then examined the proportion of participation of hospital staff members as a surgeon, first assistant, or second assistant in the operations.

\section{Microbiological surveillance}

All faculty members and surgical residents who had direct contact with patients in the hepatobiliopancreas division were screened for $S$. aureus colonization via nasal and hand swabs. Methicillin-susceptible Staphylococcus aureus and MRSA were identified using standard methods. ${ }^{13}$ Operative environments were also surveyed for infectious agents. We obtained test samples for culture from various sources, including different reusable surgical instruments, reusable laparoscopic instruments, disposable laparoscopic instruments, reusable surgical drapes, and reusable surgical gowns. In addition, we further examined the sterilization status of surgical instruments several hours after they were sterilized using a high-temperature sterilizer and ethylene oxide sterilizer.

We also reviewed the hospital infection management protocol of preoperative care, such as hair removal, skin preparation, and preoperative antibiotics.

\section{Statistical analysis}

Each variable's distributional characteristics were assessed for normality. Continuous data were reported in terms of mean \pm standard deviation and/or median with interquartile range based on variance. The chi-squared test or Fisher's exact test was used to compare categorical variables, and independent t-test or Mann-Whitney test was used to compare continuous variables. The $p$-value was adjusted under Bonferroni correction after Mann-Whitney test. A 1:3 propensity score (PS) matching between cohorts with SSI and without SSI was applied for patients' age, sex, body mass index (BMI), co-morbid diseases, operation time, laparoscopic surgery, and operation field using the nearest-neighboring matching method with $\mathrm{R}$ software (version 3.4) "MatchIt" package. ${ }^{14}$

SSI risk factor analysis was performed in the univariate logistic regression analysis and variables with $p$-value $<0.1$ 
were analyzed in the multivariate logistic regression method. Statistical analyses were performed using SPSS (version 20.0 for Mac, SPSS Inc., Chicago, IL, USA). The institutional review board of the ethics committees (IRB) of Korea University Ansan Hospital approved the study protocol. Written informed consent was waived by the IRB owing to the study's retrospective nature.

\section{RESULTS}

\section{Clinical characteristics}

Between January and February 2009, 102 patients underwent operations in the hepatobiliopancreas surgery division. Among 102 patients, SSIs occurred in 8 patients, which demonstrated showed $S$. aureus with same antibiotic susceptibility in wound culture. Operation class in SSI group was all clean-contaminated. Six cases were superficial SSI and two cases were deep SSI. Deep SSI showed

Table 1. Comparison of clinical data between patients with and those without surgical site infection before and after propensity score matching

\begin{tabular}{|c|c|c|c|c|c|c|}
\hline & \multicolumn{3}{|c|}{ Before PS matching } & \multicolumn{3}{|c|}{ After PS matching } \\
\hline & SSI $(n=8)$ & Non-SSI (n=94) & $p$-value & SSI $(n=8)$ & Non-SSI $(n=24)$ & $p$-value \\
\hline Age (years) & $\begin{array}{c}55.5 \pm 11.2 \\
57.5[23.0]\end{array}$ & $\begin{array}{c}48.6 \pm 18.3 \\
52.0[25.0]\end{array}$ & 0.293 & $55.5 \pm 11.2$ & $54.2 \pm 18.6$ & 0.674 \\
\hline Sex (male:female) & $4: 4$ & $50: 44$ & $>0.99$ & $4: 4$ & 13:11 & $>0.99$ \\
\hline BMI $\left(\mathrm{kg} / \mathrm{m}^{2}\right)$ & $\begin{array}{c}25.0 \pm 3.4 \\
24.8[5.5]\end{array}$ & $\begin{array}{c}24.2 \pm 4.0 \\
24.4[4.9]\end{array}$ & 0.520 & $25.0 \pm 3.4$ & $24.9 \pm 2.9$ & 0.948 \\
\hline \multicolumn{7}{|l|}{ Co-morbidity } \\
\hline Hypertension & $1(12.5)$ & $23(24.5)$ & 0.677 & $1(12.5)$ & $9(37.5)$ & 0.380 \\
\hline Diabetes & $1(12.5)$ & $16(17.2)$ & $>0.99$ & $1(12.5)$ & $3(12.5)$ & $>0.99$ \\
\hline Pulmonary disease & $2(25.0)$ & $5(5.3)$ & 0.093 & $2(25.0)$ & $1(4.2)$ & 0.147 \\
\hline Hepatitis & $2(25.0)$ & $12(12.8)$ & 0.302 & $2(25.0)$ & $3(12.5)$ & 0.578 \\
\hline Cancer history & $2(25.0)$ & $9(9.6)$ & 0.206 & $2(25.0)$ & $3(12.5)$ & 0.578 \\
\hline Smoking history & $2(25.0)$ & $23(24.5)$ & $>0.99$ & $2(25.0)$ & $6(25.0)$ & $>0.99$ \\
\hline Operation class & & & $>0.99$ & & & $>0.99$ \\
\hline Clean & $0(0)$ & $4(4.3)$ & & $0(0)$ & $0(0)$ & \\
\hline Clean-contaminated & $8(100)$ & $84(89.4)$ & & $8(100)$ & $22(91.7)$ & \\
\hline Contaminated & $0(0)$ & $6(6.4)$ & & $0(0)$ & $2(8.3)$ & \\
\hline Operation field & & & $0.020^{*}$ & & & 0.601 \\
\hline Liver & $2(25.0)$ & $13(13.9)$ & & $2(25.0)$ & $5(20.8)$ & \\
\hline GB/bile duct & $3(37.5)$ & $52(55.3)$ & & $3(37.5)$ & $8(33.3)$ & \\
\hline Pancreas/duodenum & $3(37.5)$ & $6(6.4)$ & & $3(37.5)$ & $5(20.8)$ & \\
\hline Others & $0(0)$ & $23(24.5)$ & & $0(0)$ & $6(25.0)$ & \\
\hline Elective operation & $8(100)$ & $69(73.4)$ & 0.194 & $8(100)$ & $17(70.8)$ & 0.150 \\
\hline General anesthesia & $8(100)$ & $91(96.8)$ & 0.608 & $8(100)$ & $23(95.8)$ & $>0.99$ \\
\hline Cancer-related Op & $6(75.0)$ & $20(21.3)$ & $0.003^{*}$ & $6(75.0)$ & $11(45.8)$ & 0.229 \\
\hline Laparoscopy & $1(12.5)$ & $60(63.8)$ & $0.007 *$ & $1(12.5)$ & $4(16.7)$ & $>0.99$ \\
\hline Op Room & & & $0.004 *$ & & & 0.414 \\
\hline 1 & $6(75.0)$ & $18(19.1)$ & & $6(75.0)$ & $11(45.8)$ & \\
\hline 2 & $1(12.5)$ & $28(29.8)$ & & $1(12.5)$ & $4(16.7)$ & \\
\hline Others & $1(12.5)$ & $48(51.1)$ & & $1(12.5)$ & $9(37.5)$ & \\
\hline Op time (minutes) & $265 \pm 91$ & $116 \pm 141$ & $<0.001 *$ & $265 \pm 91$ & $243 \pm 229$ & 0.207 \\
\hline & $271[193]$ & $75[80]$ & & $271[193]$ & $190[271]$ & \\
\hline Hospital stay (days) & $27.0 \pm 13.8$ & $16.7 \pm 40.6$ & $0.002 *$ & $27.0 \pm 13.8$ & $21.5 \pm 16.5$ & 0.214 \\
\hline & $29.0[16.0]$ & $6.5[13.0]$ & & $29.0[16.0]$ & $16.0[25.0]$ & \\
\hline
\end{tabular}

Values are presented as mean \pm standard deviation, median [interquartile range], or $\mathrm{n}(\%)$

BMI, body mass index; GB, gallbladder; MRSA, methicillin-resistant Staphylococcus aureus; Op, operation; PS, propensity score; SSI, surgical site infection

*Statistically significant 
fascia defects in wound, which were repaired with reoperation.

We compared the clinical data between the group of pa- tients with SSIs (SSI group) and the patients without SSIs (non-SSI group) before and after PS matching (Table 1). SSI group tended to be older ( $p=0.293$ ), had variable op-

Table 2. Comparison of surgeons' factors between patients with and those without surgical site infection before and after propensity score matching

\begin{tabular}{|c|c|c|c|c|c|c|}
\hline & \multicolumn{3}{|c|}{ Before PS matching } & \multicolumn{3}{|c|}{ After PS matching } \\
\hline & SSI $(n=8)$ & Non-SSI (n=94) & $p$-value & SSI $(n=8)$ & Non-SSI $(n=24)$ & $p$-value \\
\hline As an operator & & & $0.037 *$ & & & 0.803 \\
\hline A & $7(87.5)$ & $35(37.2)$ & & $7(87.5)$ & $19(79.2)$ & \\
\hline $\mathrm{B}$ & $1(12.5)$ & $41(43.6)$ & & $1(12.5)$ & $2(8.3)$ & \\
\hline $\mathrm{C}$ & $0(0)$ & $18(19.1)$ & & $0(0)$ & $3(12.5)$ & \\
\hline As an operator & & & $0.008^{*}$ & & & $>0.99$ \\
\hline A & $7(87.5)$ & $35(37.2)$ & & $7(87.5)$ & $19(79.2)$ & \\
\hline Others & $1(12.5)$ & $59(62.8)$ & & $1(12.5)$ & $5(20.8)$ & \\
\hline As a first assistant & & & 0.279 & & & 0.544 \\
\hline B & $0(0)$ & $2(2.2)$ & & $0(0)$ & $2(8.7)$ & \\
\hline $\mathrm{C}$ & $7(87.5)$ & $27(29.7)$ & & $7(87.5)$ & $12(52.2)$ & \\
\hline $\mathrm{D}$ & $0(0)$ & $12(13.2)$ & & $0(0)$ & $0(0)$ & \\
\hline $\mathrm{E}$ & $0(0)$ & $17(18.7)$ & & $0(0)$ & $5(21.7)$ & \\
\hline $\mathrm{F}$ & $0(0)$ & $7(7.7)$ & & $0(0)$ & $1(4.3)$ & \\
\hline G & $0(0)$ & $8(8.8)$ & & $0(0)$ & $0(0)$ & \\
\hline $\mathrm{H}$ & $0(0)$ & $1(1.1)$ & & $0(0)$ & $1(4.3)$ & \\
\hline I & $0(0)$ & $6(6.6)$ & & $0(0)$ & $1(4.3)$ & \\
\hline Others & $1(12.5)$ & $11(12.1)$ & & $1(12.5)$ & $1(4.3)$ & \\
\hline As a first assistant & & & $0.002 *$ & & & 0.101 \\
\hline $\mathrm{C}$ & $7(87.5)$ & $27(28.7)$ & & $7(87.5)$ & $12(50.0)$ & \\
\hline Others & $1(12.5)$ & $67(71.3)$ & & $1(12.5)$ & $12(50.0)$ & \\
\hline As a second assistant & & & $<0.001 *$ & & & $0.002 *$ \\
\hline $\mathrm{D}$ & $6(75.0)$ & $8(8.5)$ & & $6(75.0)$ & $2(8.3)$ & \\
\hline $\mathrm{E}$ & $1(12.5)$ & $3(3.2)$ & & $1(12.5)$ & $1(4.2)$ & \\
\hline $\mathrm{F}$ & $0(0)$ & $15(16.0)$ & & $0(0)$ & $6(25.0)$ & \\
\hline G & $0(0)$ & $3(3.2)$ & & $0(0)$ & $1(4.2)$ & \\
\hline $\mathrm{H}$ & $0(0)$ & $3(3.2)$ & & $0(0)$ & $0(0)$ & \\
\hline I & $0(0)$ & $0(0)$ & & $0(0)$ & $2(8.3)$ & \\
\hline Others & $1(12.5)$ & $62(66.0)$ & & $1(12.5)$ & $12(50.0)$ & \\
\hline As a second assistant & & & $<0.001 *$ & & & $0.001 *$ \\
\hline $\mathrm{D}$ & $6(75.0)$ & $8(8.5)$ & & $6(75.0)$ & $2(8.3)$ & \\
\hline Others & $2(25.0)$ & $86(91.5)$ & & $2(25.0)$ & $22(91.7)$ & \\
\hline \multicolumn{7}{|l|}{ All surgeon } \\
\hline MRSA nasal carrier & $\begin{array}{c}0.75 \pm 0.46 \\
1.0[0.75]\end{array}$ & $\begin{array}{c}0.54 \pm 0.50 \\
1.0[1.0]\end{array}$ & 0.259 & $\begin{array}{l}0.75 \pm 0.46 \\
1.0[0.75]\end{array}$ & $\begin{array}{l}0.54 \pm 0.51 \\
1.0[1.0]\end{array}$ & 0.510 \\
\hline MRSA hand carrier & $\begin{array}{c}0.75 \pm 0.46 \\
1.0[0.8]\end{array}$ & $\begin{array}{c}0.66 \pm 0.50 \\
1.0[1.0]\end{array}$ & 0.600 & $\begin{array}{l}0.75 \pm 0.46 \\
1.0[0.75]\end{array}$ & $\begin{array}{l}0.63 \pm 0.58 \\
1.0[1.0]\end{array}$ & 0.306 \\
\hline \multicolumn{7}{|l|}{ As a first assistant } \\
\hline MRSA nasal carrier & $0(0)$ & $25(26.6)$ & 0.194 & $0(0)$ & $5(20.8)$ & 0.296 \\
\hline MRSA hand carrier & $0(0)$ & $33(35.1)$ & 0.051 & $0(0)$ & $2(8.3)$ & $>0.99$ \\
\hline \multicolumn{7}{|l|}{ As a second assistant } \\
\hline MRSA nasal carrier & $6(75.0)$ & $26(27.7)$ & $0.011 *$ & $6(75.0)$ & $11(45.8)$ & 0.229 \\
\hline MRSA hand carrier & $6(75.0)$ & $29(30.9)$ & $0.019^{*}$ & $6(75.0)$ & $10(41.7)$ & 0.220 \\
\hline
\end{tabular}

Values are presented as mean \pm standard deviation, median [interquartile range], or $\mathrm{n}(\%)$

Surgeons were identified as A, B, C, D, E, F, G, H, and I

MRSA, methicillin-resistant Staphylococcus aureus; PS, propensity score; SSI, surgical site infection

*Statistically significant 
eration fields $(p=0.020)$, more cancer-related operation ( $p=0.003)$, less laparoscopic surgery $(p=0.007)$, performed in operation room $1(p=0.004)$, prolonged operation time $(p<0.001)$ and had longer hospital stays $(p=0.002)$. After PS matching, there were no statistically significant differences between two groups.

We compared surgeons' factors between the SSI and non-SSI groups (Table 2). In the SSI group, surgeon A performed operation significantly $(p=0.008)$, surgeon $\mathrm{C}$ participated operation as a first assistant significantly ( $p=$ 0.002 ), and surgeon D participated operation as a second assistant significantly $(p<0.001)$. The MRSA nasal and hand carriage of second assistant was found to be significantly higher in the SSI group ( $p=0.011$ and $p=0.019$ ). After PS matching, there was the only significant difference in the participation of surgeon $\mathrm{D}$ as a second assistant $(p=0.001)$.

\section{Results of infectious surveillance}

Nasal swab culture and hand culture of samples ob- tained from the surgical team were carried out (Table 3). Nasal swab cultures yielded three cases of MRSA infection (D, F, I) and one case of methicillin-susceptible Staphylococcus aureus infection (E). Three cases of MRSA infection (D, F, I) were detected via hand cultures. Quality control and cultures from the surgical environment, including surgical instruments, surgical drapes, and high temperature and ethylene oxide gas disinfection sterilizers, yielded no growths. The hospital infection management protocol of preoperative care, such as hair removal, skin preparation, and preoperative antibiotics has been well controlled according to the infection protocol.

\section{Outbreak control measures}

Lectures for infection prevention education were provided in the hepatobiliopancreas division between May and July 2009. During these lectures, routine practices (Standard Precautions) with an emphasis on hand hygiene were prioritized. Proper intervals for preoperative antimicrobial prophylaxis and avoidable movement in and out of the op-

Table 3. Culture results of samples obtained from surgical staffs

\begin{tabular}{|c|c|c|c|c|c|c|c|c|c|c|c|}
\hline \multirow{2}{*}{ Surgeon } & \multirow{2}{*}{ Culture } & \multirow{2}{*}{ Organism } & \multicolumn{8}{|c|}{ Antibiotic susceptibility } & \multirow{2}{*}{$\underset{\text { treatment }^{\dagger}}{\text { Organism after }}$} \\
\hline & & & PG & $\mathrm{OX}$ & VA & $\mathrm{TC}$ & EM & CI & GM & ST & \\
\hline & Wound* & S. aureus & $\mathrm{R}$ & $\mathrm{R}$ & $\mathrm{S}$ & S & S & S & S & S & \\
\hline A & $\begin{array}{l}\text { Nasal swab } \\
\text { Hand }\end{array}$ & & & & & & & & & & \\
\hline B & $\begin{array}{l}\text { Nasal swab } \\
\text { Hand }\end{array}$ & & & & & & & & & & \\
\hline $\mathrm{C}$ & $\begin{array}{l}\text { Nasal swab } \\
\text { Hand }\end{array}$ & & & & & & & & & & \\
\hline $\mathrm{D}$ & Nasal swab & S. aureus & $\mathrm{R}$ & $\mathrm{R}$ & S & S & S & $\mathrm{S}$ & S & S & - \\
\hline & Hand & S. aureus & $\mathrm{R}$ & $\mathrm{R}$ & $\mathrm{S}$ & S & S & $\mathrm{S}$ & S & S & S. aureus \\
\hline $\mathrm{E}$ & $\begin{array}{l}\text { Nasal swab } \\
\text { Hand }\end{array}$ & S. aureus & $\mathrm{R}$ & $\mathrm{S}$ & $\mathrm{S}$ & $\mathrm{S}$ & $\mathrm{S}$ & $\mathrm{S}$ & $\mathrm{S}$ & $\mathrm{S}$ & - \\
\hline $\mathrm{F}$ & Nasal swab & S. aureus & $\mathrm{R}$ & $\mathrm{R}$ & $\mathrm{S}$ & S & S & $\mathrm{S}$ & S & $\mathrm{S}$ & - \\
\hline & Hand & S. aureus & $\mathrm{R}$ & $\mathrm{R}$ & S & S & $\mathrm{S}$ & $\mathrm{S}$ & S & $\mathrm{S}$ & - \\
\hline G & Nasal swab & & & & & & & & & & \\
\hline & Hand & S. aureus & $\mathrm{R}$ & $\mathrm{R}$ & $\mathrm{S}$ & S & $\mathrm{S}$ & $\mathrm{S}$ & S & $\mathrm{S}$ & - \\
\hline $\mathrm{H}$ & $\begin{array}{l}\text { Nasal swab } \\
\text { Hand }\end{array}$ & & & & & & & & & & \\
\hline I & Nasal swab & S. aureus & $\mathrm{R}$ & $\mathrm{R}$ & $\mathrm{S}$ & S & $\mathrm{S}$ & $\mathrm{S}$ & $\mathrm{S}$ & $\mathrm{S}$ & - \\
\hline & Hand & S. aureus & $\mathrm{R}$ & $\mathrm{R}$ & $\mathrm{S}$ & S & $\mathrm{S}$ & $\mathrm{S}$ & $\mathrm{S}$ & $\mathrm{S}$ & - \\
\hline
\end{tabular}

Surgeons were identified as A, B, C, D, E, F, G, H, and I

CI, ciprofloxacin; EM, erythromycin; GM, gentamicin; OX, oxacillin; PG, penicillin G; R, resistant; S, susceptible; ST, sulfamethoxazole/trimethoprim; TC, teicoplanin; VA, vancomycin

* Surgical site showed S. aureus with same antibiotic susceptibility

${ }^{\dagger}$ Mupirocin ointment and rifampin medication 
Table 4. Regression analysis of significant risk factors for surgical site infections in the univariate and multivariate analysis before and after propensity score matching

\begin{tabular}{|c|c|c|c|c|}
\hline & \multicolumn{2}{|c|}{ Univariate analysis } & \multicolumn{2}{|c|}{ Multivariate analysis } \\
\hline & $p$-value & HR $(95 \%$ CI $)$ & $p$-value & $\operatorname{HR}(95 \% \mathrm{CI})$ \\
\hline \multicolumn{5}{|c|}{ Before PS matching } \\
\hline Age & 0.298 & $1.025(0.979-1.072)$ & & \\
\hline Male sex & 0.862 & $1.136(0.268-4.815)$ & & \\
\hline $\mathrm{A}$ as an operator & 0.054 & $0.122(0.014-1.040)$ & 0.354 & $0.233(0.011-5.062)$ \\
\hline $\mathrm{C}$ as a first assistant & $0.009^{*}$ & $17.370(2.039-147.992)$ & 0.230 & $3.519(0.115-108.096)$ \\
\hline $\mathrm{D}$ as a second assistant & $<0.001^{*}$ & $32.250(5.567-186.824)$ & 0.998 & $0.161(0.008-3.180)$ \\
\hline Open surgery & $0.021 *$ & $12.353(1.458-104.690)$ & 0.429 & $3.127(0.186-52.607)$ \\
\hline Cancer-related operation & $0.005^{*}$ & $11.100(2.080-59.249)$ & 0.447 & $0.393(0.035-4.396)$ \\
\hline Operation time & $0.034^{*}$ & $1.004(1.000-1.008)$ & 0.441 & $1.002(0.997-1.008)$ \\
\hline Nasal MRSA carrier ${ }^{\dagger}$ & $0.015^{*}$ & $0.127(0.024-0.672)$ & $>0.99$ & \\
\hline 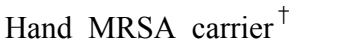 & $0.024 *$ & $0.149(0.028-0.781)$ & $>0.99$ & \\
\hline \multicolumn{5}{|c|}{ After PS matching } \\
\hline Age & 0.663 & $1.010(0.965-1.058)$ & & \\
\hline Male sex & 0.838 & $1.182(0.238-5.864)$ & & \\
\hline A as an operator & 0.605 & $1.842(0.182-18.657)$ & & \\
\hline $\mathrm{C}$ as a first assistant & 0.089 & $7.000(0.743-65.943)$ & 0.361 & $3.423(0.244-47.977)$ \\
\hline $\mathrm{D}$ as a second assistant & $0.001 *$ & $33.000(3.814-285.533)$ & $0.004 *$ & $25.088(2.759-228.149)$ \\
\hline Open surgery & 0.779 & $1.400(0.133-14.743)$ & & \\
\hline Cancer-related operation & 0.166 & $3.545(0.592-21.245)$ & & \\
\hline Operation time & 0.707 & $1.001(0.997-1.005)$ & & \\
\hline Nasal MRSA carrier ${ }^{\dagger}$ & 0.117 & $4.200(0.698-25.264)$ & & \\
\hline Hand MRSA carrier ${ }^{\dagger}$ & 0.166 & $0.282(0.047-1.690)$ & & \\
\hline
\end{tabular}

Surgeons were identified as A, B, C, D, E, F, G, H, and I

PSM, propensity score matching; HR, hazard ratio; CI, confidence interval

*Statistically significant

${ }^{\dagger}$ Participation as a second assistant

erating room was also reinforced. The staffs were made aware of the constant transmission of MRSA.

Hospital members with nasal MRSA colonization were decolonized with mupirocin ointment ( 2 times per day) and rifampin $600 \mathrm{mg}$ (once a day) for 5 days. Hospital members with hand MRSA colonization were decolonized via a $4 \%$ chlorhexidine shower twice. Surveillance for Staphylococcus infections was followed up 4 weeks after the $S$. aureus decolonization, and only one case of Staphylococcus colonization detected via a hand culture was found during the process (surgeon D, Table 3).

\section{Analysis of risk factors affecting the outbreak of MRSA-related SSI}

In univariate analysis before PS matching, surgeon $\mathrm{C}$ as a first assistant $\mathrm{C}(p=0.009)$, surgeon $\mathrm{D}$ as a second assistant $(p<0.001)$, open surgery $(p=0.021)$, cancer-re- lated operation $(p=0.005)$, longer operation time $(p=0.034)$, MRSA nasal carriage $(p=0.015)$, and MRSA hand carriage $(p=0.024)$ were significant factors for SSI in our study (Table 4). In the multivariate analysis before PS matching, there was no significant risk factor for MRSA-related SSI. After propensity score matching, surgeon D as a second assistant was the only significant risk factor for MRSA SSI in univariate $(p=0.001)$ and multivariate analysis $(p=$ 0.004 , hazard ratio $=25.088,95 \%$ confidence interval $=2.759$ 228.149).

\section{DISCUSSION}

This observational, retrospective, and epidemiologic study was conducted to establish the origin of a $S$. aureus outbreak, which was eventually identified via detection of nasal and hand MRSA carriage in a healthcare worker, 
a surgeon in particular. The exact position where the dissemination occurred was, however, not recognized. While some reports propose that healthcare workers with MRSA carriage are infection targets rather than offenders of outbreaks, ${ }^{15}$ other reports have focused on the significance of MRSA carriage in healthcare workers in SSI outbreaks. ${ }^{16,17}$ In our study, surgeon D had hand and nose MRSA carriage, and the colonies could not be completely eradicated even after the medication. This indicates the possibility of suboptimal treatment compliance of surgeon D in terms of the prevention of SSI, which intensified the degree of SSIs.

Devenish and Miles ${ }^{18}$ have proposed that the operators' or assistants' hand S. aureus carriage could infect the surgical site via breaks in surgical gloves. Another study demonstrated that nasal and hand S. aureus colonies played a significant role in surgical site infection outbreak. A high proportion of the surgeons in the operating environment with MRSA carriage could indicate dispersion among healthcare workers. ${ }^{19}$ In our study, the mean operation time in SSI group was $265 \pm 91$ minutes, which meant that the probability of defects to the surgical gloves became increased. Makama et al. ${ }^{20}$ have demonstrated that the longer the duration of operation, the greater the risk of surgical glove perforation in the randomized controlled study. They also reported that second assistant had a higher perforation rate of surgical glove. At the end of the operation, fascia closure suturing was mainly performed by the first and second assistant, and skin suturing was performed primarily by the second assistant in our institute. A high rate of colonization found in healthcare staff during a $S$. aureus outbreak was reported by Weber et al., ${ }^{19}$ and the outbreak was thought to have occurred because of the presence of virulent colonizing considerations in the outbreak strains and the spread of the strain due to diffusion from people (not recognized in our study). In our study, suboptimal compliance under standard regulations of infection control was a liable causative source in the spread of the outbreak strains, which could be deduced via the positive hand culture for MRSA after treatment in surgeon D.

On the other hand, many studies have focused on the MRSA nasal carriage of patients as the source of SSIs. The most widely described risk factor specific to SSI due to $S$. aureus is nasal colonization in patients. ${ }^{21}$ The risk of $S$. aureus nosocomial infections is greater in preopera- tive carriers than in non-carriers, and almost patients who develop SSIs already have an MRSA colonization. Patients with nasal MRSA carriage are at greater risk for nosocomial $S$. aureus bacteremia than control groups. ${ }^{22}$ However, this is additionally understandable from the previous documented data, wherein preoperative nasal or hand carriage does not explain all of the SSIs. ${ }^{23}$ Given that routine preoperative screening for $S$. aureus in patients was not conducted in our study, we could not assess this risk factor. However, the potential benefits and harms of using decontamination for the prevention of SSI remain uncertain. $^{24}$

Our study confirmed that nasal colonization of MRSA in a surgeon is an important factor for the development of MRSA SSI in the perioperative period of hepatobiliopancreatic surgery. The specific strain of MRSA found in both the patients and surgeon D demonstrated identical result of antibiotic susceptibilities. After extermination of the strain obtained from the surgeon with MRSA carriage, we could manage the SSI outbreak and no additional SSIs were detected. There is some evidence to suggest that the screening of healthcare workers, in principle, acceptable to both patients and healthcare workers. However, evidence regarding its effectiveness in the prevention and control of MRSA in the endemic setting is limited. ${ }^{11}$

The optimal antibiotic prophylactic regimen for MRSArelated SSI prevention has not been identified thus far. We currently have no evidence to confirm that preoperative administration of multiple antibiotics or long-term antibiotics administration is beneficial for controlling and preventing MRSA-related SSIs. ${ }^{25}$

We also analyzed other risk factors for SSIs due to MRSA. Longer operation duration is a well-known perioperative risk factor for $\mathrm{SSI}^{26}$ and for $S$. aureus-related SSIs. ${ }^{21}$ Cancer-related surgery was also identified as a significant risk factor for SSI. ${ }^{26}$ However, in this study, longer operation duration and cancer-related surgery showed no significant differences in terms of incidence of SSI, despite the fact that those factors were significant in the univariate analysis before propensity score matching. This difference could be associated with a difference in patient populations, surgical field, as well as our somewhat smaller population size.

Such outbreaks can be prevented by a combination of infection control practices: isolation of affected patients; 
importance on hand hygiene; improved environmental disinfection with a hypochlorite agent; and screening for MRSA colonization via nasal and hand cultures. Supplementary education and teaching were also provided in the surgery department after the outbreak of MRSA.

This study has some limitations. We could not confirm preoperative $S$. aureus colonization status in our patients, because this study was retrospective in nature. The relatively small number of infection cases might have decreased our capacity to identify significant risk factors despite of PS matching statistical analysis. Moreover, we did not perform genetic testing of the $S$. aureus strains isolated during the outbreak and that obtained from surgeon D. We believe that the outbreak strain was originated from surgeon D because of the same antibiotic susceptibility. This study was conducted in the hepatobiliopancreas surgery division of a single university hospital, and the SSI outbreak epidemiological study results might be applicable to in-hospital patients undergoing hepatobiliopancreas surgeries elsewhere as well. Furthermore, the feasibility of preoperative additive screening for S. aureus and decolonizing surgeons is being explored within the hepatobiliopancreas surgery division.

In conclusion, the SSI outbreak in the hepatobiliopancreas surgery division occurred due to transmission of MRSA from a surgeon to patients in the setting of standard perioperative infection control measures, which did not include preoperative screening of MRSA for surgeons. The outbreak was associated with a high prevalence of nasal and hand carriage of the outbreak MRSA strain among the surgeons, especially who participated in surgery as a second assistant. We recommend the preoperative regular nasal and hand screening of surgeons to prevent the spread of MRSA.

\section{ACKNOWLEDGEMENTS}

The work was supported by the research seed fund from the Korea University Research and Business Foundation.

\section{REFERENCES}

1. Hidron AI, Edwards JR, Patel J, Horan TC, Sievert DM, Pollock DA, et al.; National Healthcare Safety Network Team; Participating National Healthcare Safety Network Facilities. NHSN annu- al update: antimicrobial-resistant pathogens associated with healthcare-associated infections: annual summary of data reported to the National Healthcare Safety Network at the Centers for Disease Control and Prevention, 2006-2007. Infect Control Hosp Epidemiol 2008;29:996-1011.

2. Diekema D, Johannsson B, Herwaldt L, Beekmann S, Jernigan $\mathrm{J}$, Kallen A, et al. Current practice in Staphylococcus aureus screening and decolonization. Infect Control Hosp Epidemiol 2011;32:1042-1044.

3. Peterson LR, Liesenfeld O, Woods CW, Allen SD, Pombo D, Patel PA, et al. Multicenter evaluation of the LightCycler methicillin-resistant Staphylococcus aureus (MRSA) advanced test as a rapid method for detection of MRSA in nasal surveillance swabs. J Clin Microbiol 2010;48:1661-1666.

4. Kim MH, Lee WI, Kang SY. Detection of methicillin-resistant Staphylococcus aureus in healthcare workers using real-time polymerase chain reaction. Yonsei Med J 2013;54:1282-1284.

5. Safdar N, Bradley EA. The risk of infection after nasal colonization with Staphylococcus aureus. Am J Med 2008;121:310315.

6. Solberg CO. Spread of Staphylococcus aureus in hospitals: causes and prevention. Scand J Infect Dis 2000;32:587-595.

7. Eveillard M, Martin Y, Hidri N, Boussougant Y, Joly-Guillou ML. Carriage of methicillin-resistant Staphylococcus aureus among hospital employees: prevalence, duration, and transmission to households. Infect Control Hosp Epidemiol 2004;25:114-120.

8. Albrich WC, Harbarth S. Health-care workers: source, vector, or victim of MRSA? Lancet Infect Dis 2008;8:289-301.

9. Bode LG, Kluytmans JA, Wertheim HF, Bogaers D, VandenbrouckeGrauls CM, Roosendaal R, et al. Preventing surgical-site infections in nasal carriers of Staphylococcus aureus. N Engl J Med 2010;362:9-17.

10. Ayliffe GA, Lowbury EJ. Airborne infection in hospital. J Hosp Infect 1982;3:217-240.

11. Hawkins G, Stewart S, Blatchford O, Reilly J. Should healthcare workers be screened routinely for meticillin-resistant Staphylococcus aureus? A review of the evidence. J Hosp Infect 2011; 77:285-289.

12. Mangram AJ, Horan TC, Pearson ML, Silver LC, Jarvis WR. Guideline for prevention of surgical site infection, 1999. Hospital Infection Control Practices Advisory Committee. Infect Control Hosp Epidemiol. 1999;20:250-278; quiz 279-280.

13. Murray PR, Baron EJ, Jorgensen JH, Landry ML, Pfaller MA, Yolken RH. Manual of clinical microbiology. Washington: American Society of Microbiology, 1999.

14. Zhang Z. Propensity score method: a non-parametric technique to reduce model dependence. Ann Transl Med 2017;5:7.

15. Blok HE, Troelstra A, Kamp-Hopmans TE, Gigengack-Baars AC, Vandenbroucke-Grauls CM, Weersink AJ, et al. Role of healthcare workers in outbreaks of methicillin-resistant Staphylococcus aureus: a 10-year evaluation from a Dutch university hospital. Infect Control Hosp Epidemiol 2003;24:679-685.

16. Lin YC, Lauderdale TL, Lin HM, Chen PC, Cheng MF, Hsieh $\mathrm{KS}$, et al. An outbreak of methicillin-resistant Staphylococcus aureus infection in patients of a pediatric intensive care unit and high carriage rate among health care workers. J Microbiol Immunol Infect 2007;40:325-334.

17. Sassmannshausen R, Deurenberg RH, Köck R, Hendrix R, Jurke A, Rossen JW, et al. MRSA prevalence and associated risk factors among health-care workers in non-outbreak situations in the Dutch-German EUREGIO. Front Microbiol 2016;7:1273.

18. Devenish EA, Miles AA. Control of Staphylococcus aureus in an operating-theatre. Lancet 1939;223:1088-1094.

19. Weber S, Herwaldt LA, Mcnutt LA, Rhomberg P, Vaudaux P, 
Pfaller MA, et al. An outbreak of Staphylococcus aureus in a pediatric cardiothoracic surgery unit. Infect Control Hosp Epidemiol 2002;23:77-81.

20. Makama JG, Okeme IM, Makama EJ, Ameh EA. Glove perforation rate in surgery: a randomized, controlled study to evaluate the efficacy of double gloving. Surg Infect (Larchmt) 2016;17: 436-442.

21. Muñoz P, Hortal J, Giannella M, Barrio JM, Rodríguez-Créixems $\mathrm{M}$, Pérez MJ, et al. Nasal carriage of $\mathrm{S}$. aureus increases the risk of surgical site infection after major heart surgery. J Hosp Infect 2008;68:25-31.

22. Jensen AG, Wachmann CH, Poulsen KB, Espersen F, Scheibel J, Skinhøj P, et al. Risk factors for hospital-acquired Staphylococcus aureus bacteremia. Arch Intern Med 1999;159:1437-1444.
23. Herwaldt LA. Staphylococcus aureus nasal carriage and surgical-site infections. Surgery 2003;134(5 Suppl):S2-S9.

24. Liu Z, Norman G, Iheozor-Ejiofor Z, Wong JK, Crosbie EJ, Wilson P. Nasal decontamination for the prevention of surgical site infection in Staphylococcus aureus carriers. Cochrane Database Syst Rev 2017;5:CD012462.

25. Gurusamy KS, Koti R, Wilson P, Davidson BR. Antibiotic prophylaxis for the prevention of methicillin-resistant Staphylococcus aureus (MRSA) related complications in surgical patients. Cochrane Database Syst Rev 2013;(8):CD010268.

26. Cheng K, Li J, Kong Q, Wang C, Ye N, Xia G. Risk factors for surgical site infection in a teaching hospital: a prospective study of 1,138 patients. Patient Prefer Adherence 2015;9:11711177. 